\title{
The Correlation Between Electrodiagnostic Results and Ultrasonographic Findings in the Severity of Carpal Tunnel Syndrome in Females
}

\author{
Da Sol Ha, MD, Hyoung Seop Kim, MD, Jong Moon Kim, MD, Kun Hee Lee, MD
}

Department of Physical Medicine and Rehabilitation, National Health Insurance Service Ilsan Hospital, Goyang, Korea

\begin{abstract}
Objective To determine which ultrasonographic measurement can be used as an indicator reflecting the severity of carpal tunnel syndrome (CTS), by comparing electrodiagnostic results with ultrasonographic measurements in females. Many previous studies have tried to reveal that the ultrasonography (US) can possibility be used for diagnosis and severity of CTS. However, the criteria are different by gender. Thus far, there have been many efforts towards providing patients with a CTS diagnosis and severity prediction using US, but studies' results are still unclear due to lack of data on gender differences.

Methods We collected data from 54 female patients. We classified the severity of CTS according to electrodiagnostic results. Ultrasonographic measurements included proximal and distal cross-sectional areas of the median nerve and carpal tunnel.

Results The severity by electrodiagnostic results statistically correlated to the proximal cross-sectional area (CSA) of the median nerve and carpal tunnel. However, there was no relationship between the proximal and distal nerve/tunnel indexes and the severity by electrodiagnostic results.

Conclusion In female patients with CTS, the proximal CSAs of the median nerve and carpal tunnel increase. They correlate with the severity by electrodiagnostic findings. The CSA of the proximal median nerve could be particularly used as a predictor of the severity of CTS in female patients. However, the nerve/tunnel index is constant, irrespective of the severity of CTS.
\end{abstract}

Keywords Carpal tunnel syndrome, Electrodiagnosis, Ultrasonography, Median nerve

\section{INTRODUCTION}

Carpal tunnel syndrome (CTS) is one of the most com- mon peripheral neuropathies caused by entrapment of the median nerve within the wrist joint [1-3]. An electrodiagnostic study (EDS) is a confirmative tool for the di-

Received May 18, 2016; Accepted October 12, 2016

Corresponding author: Hyoung Seop Kim

Departments of Physical Medicine and Rehabilitation, National Health Insurance Service Ilsan Hospital, 100 Ilsan-ro, Ilsandong-gu, Goyang 10444, Korea. Tel: +82-31-900-0137, Fax: +82-31-900-0343, E-mail: rehappydoc@gmail.com

ORCID: Da Sol Ha (http://orcid.org/0000-0001-6390-657X); Hyoung Seop Kim (http://orcid.org/0000-0002-5310-4802); Jong Moon Kim (http://orcid. org/0000-0002-8684-8736); Kun Hee Lee (http://orcid.org/0000-0001-7904-9840).

(c) This is an open-access article distributed under the terms of the Creative Commons Attribution Non-Commercial License (http://creativecommons.org/ licenses/by-nc/4.0) which permits unrestricted noncommercial use, distribution, and reproduction in any medium, provided the original work is properly cited. Copyright (c) 2017 by Korean Academy of Rehabilitation Medicine 
agnosis of CTS that differentiates it from other peripheral neuropathies $[3,4]$. However, using an EDS causes the patient pain, and an EDS cannot provide morphological information on the median nerve or its surroundings for determining the etiology [3].

Thus, in recent years, ultrasonography (US) developed. Its value lies in its ability to assess both the median nerve and carpal tunnel pathology $[1,5]$. US also has the advantage of being lower cost, requiring a shorter examination time, allowing for easier discrimination of etiologies, and allowing for the application of US-guided injection for the treatment $[3,4,6,7]$.

Previous studies suggest US should be applied differently between males and females. For instance, in a previous study, the sizes of the median nerve and the carpal tunnel were greater in male participants, as a result of the ultrasonographic exam [5]. Another study revealed that ultrasonographic measurements in the females showed statistically significant differences when comparing affected and non-affected hands; however, there were no such statistical differences in males [8]. Therefore, the cut-off values in cross-sectional areas (CSAs) of the median nerve and carpal tunnel should be set separately according to gender in an ultrasonographic diagnosis of CTS. The correlation of ultrasonographic measurements to electrophysiologic results should also depend on gender.

Gender may also play a role in ultrasonographic measurements and electrophysiologic severity. Some previous studies have reported on the correlation between ultrasonographic measurement and electrophysiologic severity [1,9-12]. Researchers generally agree that the CSA of the median nerve in CTS is larger than that in the normal population. In addition, some researchers advocate that there is a clinically significant correlation between the ultrasonographic measurement and the electrophysiologic severity $[1,9,10]$. However, other researchers oppose these findings, and posture that ultrasonographic measurements cannot reflect on the severity of electrodiagnosis. Rather, the ultrasonography is just a supplemental procedure for diagnosing CTS $[11,12]$. This disagreement may come from exclusion of gender from consideration. In other words, in some of these previous studies, the standards of diagnosis for severity of CTS by US usually did not consider gender differences. The failure to take into account gender may be problematic, as the normal mean value of ultrasonographic measurements in males is larger than females. Therefore, without considering gender in the ultrasonographic diagnosis of CTS, the probability of false-positive results may increase in males whereas false-negative results may increase in females [5]. However, there are only a few studies on the relationship between ultrasonographic measurement and electrophysiologic severity that take into consideration any gender differences $[13,14]$.

The aim of this study is to find the relationship between electrophysiologic severity of CTS and the ultrasonographic indexes (including CSA of the median nerve, CSA of the carpal tunnel, and nerve/tunnel index) in females, and to establish a cut-off value of ultrasonographic measurements for predicting the severity level of CTS in females.

\section{MATERIALS AND METHODS}

\section{Subjects}

We collected data from 58 female patients. These patients were clinically suspected of having CTS, visited our outpatient clinic, and presented symptoms and signs of CTS. These symptoms included palm abnormal sensation on the median nerve distribution, weakness of grasp power, atrophy of the thenar muscles, positive finding of Tinnel's sign, and Phalen's maneuver. We collected this data between March 2011 and March 2012.

An Institutional Review Board approved this study's protocol (No. 2014-11-009). We obtained informed consent from all subjects before proceeding with this study. A physiatrist took the medical history of each patient and examined them for the typical symptoms of CTS with worsening factors. Before US and EDS, the side at which symptoms manifested was recorded along with body indexes, such as height, weight, and body mass index (BMI).

We excluded patient samples with previous trauma or surgical history around the wrist; hereditary or acquired diseases that can cause peripheral neuropathy; atypical nerve or vessel shapes, such as bifid median nerve or persistent median nerve artery; and mass lesions in the wrist that can cause median nerve compression, including ganglion cysts, neurogenic tumors, flexor tenosynovitis, and accessory flexor digitorum superficialis. After this exclusion process, we had a sample size of 58 patients. We then excluded three patients because they did not 
undergo both US and EDS, and excluded one patient because her body indexes were not collected. As a result, we initially enrolled 58 patients, and then examined 108 hands of 54 female patients (mean age, 53.79 years; range, 31-82 years).

\section{Electrodiagnostic study}

All patients underwent a standardized nerve conduction study based on the American Academy of Neurology summary statement regarding the desired protocol for patients who are suspected of having CTS [15]. EDS was performed on both hands regardless of the side that symptoms manifested. Electrodiagnostic indexes included terminal latency and peak-to-peak amplitude of median motor nerve conduction study (NCS) from wrist to abductor pollicis brevis (APB) and terminal latency, peak-to-peak amplitude and velocity of orthodromic median sensory NCS from index finger to wrist. We used these electrodiagnostic indexes for a more detailed severity classification. If these two initial EDSs were normal, three more sensitive studies were undertaken: (1) orthodromic median and ulnar sensory NCS-stimulation (ring finger), recording (median nerve at wrist) [16]; (2) palm-wrist median-ulnar sensory latency comparison test-stimulation (median nerve and ulnar nerve in the palm), recording (median nerve and ulnar nerve at wrist) [17]; and (3) antidromic median sensory short segment study-stimulation (median nerve in serial $1 \mathrm{~cm}$ increments across the carpal tunnel), recording (index finger) [17].

In test (1), the ring finger was stimulated by using a ring electrode. We made a recording over the median nerve at the wrist level. If there were two distinctive peak latencies, we considered the graph a dual peak wave form and categorized this as a positive finding [16].

In test (2), we stimulated the median nerve and ulnar nerve in the palm, and conducted the recording at the wrist over the course of the median and ulnar nerve, at 8 $\mathrm{cm}$ distal of the stimulation site. An onset latency difference of $\geq 0.2 \mathrm{~ms}$ was representative of a relative slowing of the median nerve compared to ulnar across the carpal tunnel [17]. We categorized this as a positive finding.

In test (3), called the inching test, the median nerve was stimulated in serial $1 \mathrm{~cm}$ increments across the carpal tunnel (8 segments; from proximal $-1 \mathrm{~cm}$ to distal $6 \mathrm{~cm}$ of distal wrist crease). We recorded from the index finger.
We categorized a segmental peak latency difference of $\geq 0.5 \mathrm{~ms}$ as a positive finding [17].

Of these three additional tests, if we found a positive finding in two or all three tests, we diagnosed the hand with very mild CTS (score $=1$ ) [18].

We classified the results of EDS with a severity score (0-6) in line with the neurophysiologic grading scale by Bland [18]. The scoring was as follows: normal (score $=0$ ), denoting no neurophysiological abnormality; very mild CTS (score $=1$ ) detected in two or more additional three sensitive studies; mild CTS (score $=2$, orthodromic sensory conduction velocity from index finger to wrist $<40 \mathrm{~m} /$ $\mathrm{s}$ with motor terminal latency from wrist to APB $<4.5 \mathrm{~ms}$ ); moderately severe CTS (score $=3$, motor terminal latency $>4.5 \mathrm{~ms}$ and $<6.5 \mathrm{~ms}$ with preserved index finger SNAP); severe CTS (score $=4$, motor terminal latency $>4.5 \mathrm{~ms}$ and $<6.5 \mathrm{~ms}$ with absent SNAP); very severe CTS (score $=5$, motor terminal latency $>6.5 \mathrm{~ms}$ ); and extremely severe CTS (score $=6$, surface motor potential from APB $<0.2 \mathrm{mV}$, peak-to-peak). Based on this classification, a hand having a severity score of 1 to 6 was diagnosed with CTS.

We reclassified the above severity score into four severity grades: 0, I, II, and III. A severity score 0 (normal group) was grade 0 ; a severity score of 1 and 2 (very mild to mild groups of CTS) reclassified to grade I; a severity score of 3 and 4 (moderately severe to severe groups of CTS) reclassified to grade II; and a severity score of 5 and 6 (very severe to extremely severe groups of CTS) reclassified to grade III. We intended to recommend the cut-off values of US findings to discriminate the grades of severity in CTS according to these four groups.

We measured these parameters by using standard techniques of supramaximal stimulation, surface and ring electrodes (Medelec Synergy; Oxford Instruments Medical, Abingdon, UK). We conducted all tests in the same room, measured the room temperature, and maintained it between $24.0^{\circ} \mathrm{C}$ and $26.0^{\circ} \mathrm{C}$. We took the hand temperature of each patient before conducting EDS, and all the temperatures were more than $32.0^{\circ} \mathrm{C}$. The examiners for EDS had no knowledge of the ultrasonographic results, since US was performed after the EDS.

A physiatrist specializing in EDS (SW Kim) guided us in performing and interpreting the nerve conduction studies. We also had the support of trainees of physical medicine and rehabilitation. 


\section{Ultrasonographic technique}

The patients underwent US within one week after EDS. A physiatrist (HS Kim) with at least 5 years of musculoskeletal US experience performed the ultrasonographic examinations. He measured CSAs and nerve/tunnel index on both hands, regardless of the side that symptoms manifested. The physiatrist was blinded to the results of EDS. He measured the proximal and distal CSAs of both the median nerve and carpal tunnel using a 5-12 $\mathrm{MHz}$ (GE Voluson i; GE Healthcare, Waukesha, WI, USA) linear array transducer. During US, we asked the patient to lie supine with the forearm supinated, and we placed a shallow pillow underneath the wrist. To minimize sampling errors due to differential loads, we avoided pressure to the wrist during scanning and measurement.

We measured the CSAs of the median nerve and carpal tunnel at two levels: proximal and distal CSAs of the carpal tunnel were measured at the scaphoid pisiform level (Fig. 1) and at the trapezium hamate level (Fig. 2), respectively. At each level, the medial and lateral sides of the carpal bone constituted the inner boundaries of the carpal tunnel, while the flexor tendon sheath and flexor retinaculum formed the superior and inferior boundaries, respectively.

We directly calculated the CSAs of the median nerve and carpal tunnel by continuously tracing the nerve and carpal tunnel through their echogenic boundary. To minimize measurement errors, we repeated assessments three times at both levels. We used a median value for statistical evaluation.

We obtained the proximal or distal nerve/tunnel index by calculating the ratio of the proximal or distal CSA of the median nerve to that of carpal tunnel, and multiplied the value by 100 [5].

\section{Statistical analyses}

We statistically analyzed all data using SAS for Windows (Korean ver. 9.2), statistical package (SAS Institute Inc., Cary, NC, USA). We performed correlation analyses by obtaining Spearman coefficients between seven electrophysiologic severity groups and anthropometric data, and US measurements. We performed a one-way analysis
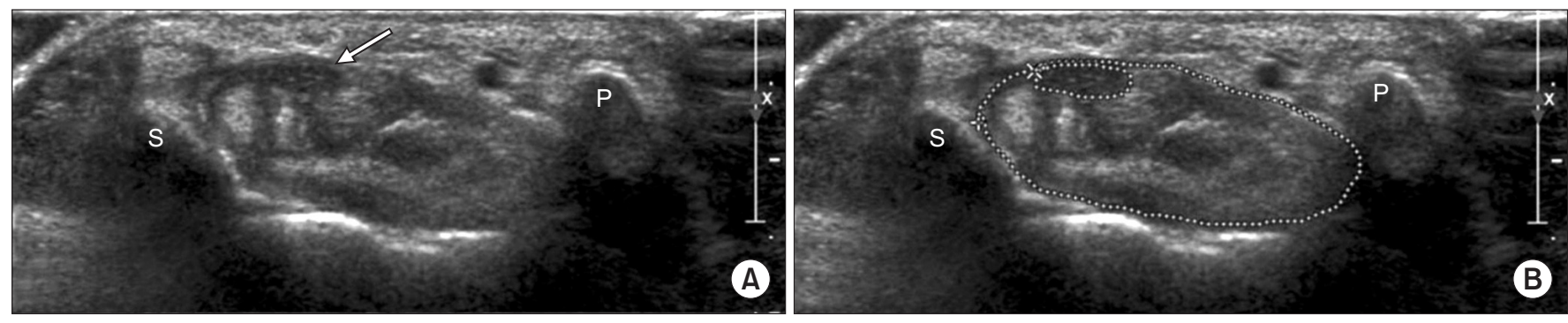

Fig. 1. Cross-sectional areas of the median nerve and carpal tunnel at proximal carpal tunnel level. (A) Proximal carpal tunnel, median nerve (arrow), and flexor tendon groups are shown between the pisiform and scaphoid bones. (B) Dotted outlines of median nerve and carpal tunnel are shown at proximal carpal tunnel level. S, means scaphoid bone; $\mathrm{P}$, means pisiform bone.
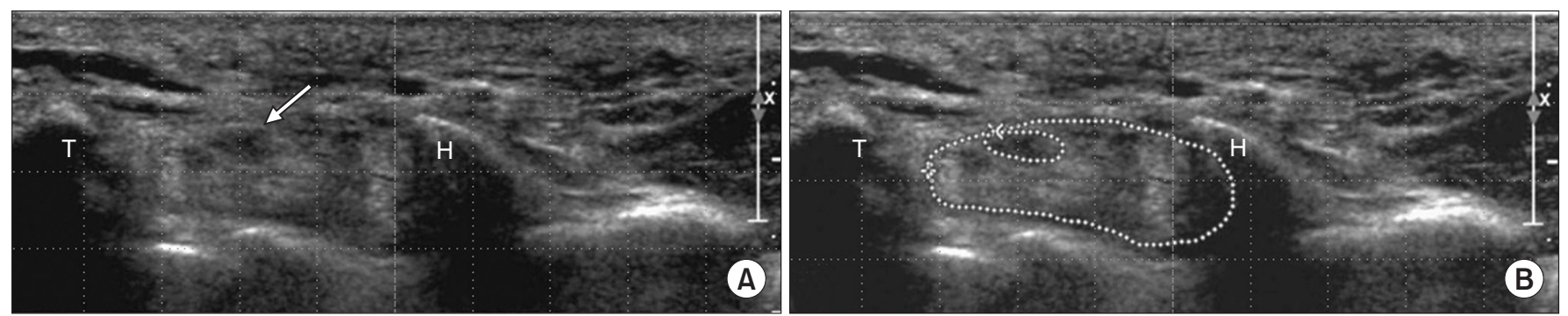

Fig. 2. Cross-sectional areas of the median nerve and carpal tunnel at distal carpal tunnel level. (A) Proximal carpal tunnel, median nerve (arrow), and flexor tendon groups are shown between the trapezium and hook of hamate bones. (B) Dotted outlines of median nerve and carpal tunnel are shown at distal carpal tunnel level. T, means trapezium bone; $\mathrm{H}$, means hook of hamate bone. 

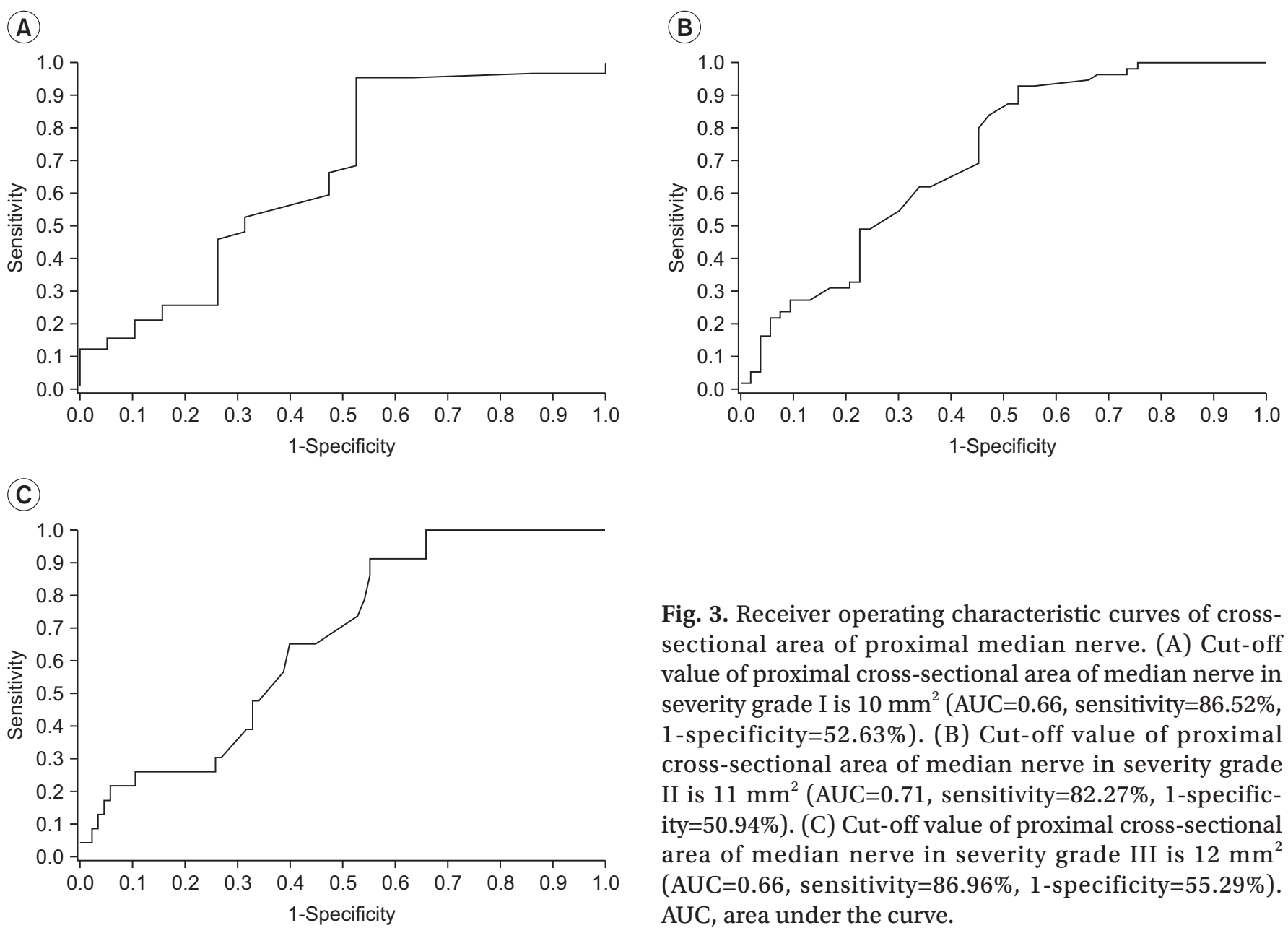

of variance tests for the comparison of anthropometric data and ultrasonographic measurements between severity grades by EDS. We used Fisher least significant difference method for our post-hoc analysis.

The standard, in diagnosis of CTS by EDS, came from the 'more than very mild CTS' group (score $\geq 1$ ) of severity grading scale from Bland [18]. To set cut-off values of ultrasonographic severity of CTS, we performed a receiver operating characteristic (ROC) analyses and maximal Youden analysis. We conducted the analyses for each severity grade standard (Fig. 3). A p-value $<0.05$ indicated statistical significance.

\section{RESULTS}

\section{General characteristics and electrodiagnostic and} ultrasonographic findings of the subjects

Mean values of age, height, body weight, and BMI of the patients were $53.79 \pm 8.64$ years, $157.25 \pm 3.88 \mathrm{~cm}$,

Table 1. Severity grade and score of carpal tunnel syndrome by electrodiagnostic study

\begin{tabular}{clrrc}
\hline $\begin{array}{c}\text { Severity } \\
\text { grade }\end{array}$ & Severity (score) & Right & Left & Total \\
\hline 0 & Normal (0) & 7 & 12 & 19 \\
\hline I & Very mild (1) & 8 & 7 & 15 \\
\hline & Mild (2) & 11 & 8 & 19 \\
\hline II & Moderately severe (3) & 16 & 12 & 28 \\
\hline \multirow{2}{*}{ III } & Severe (4) & 3 & 1 & 4 \\
\hline & Very severe (5) & 8 & 12 & 20 \\
\hline
\end{tabular}

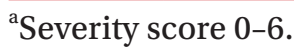

$60.03 \pm 7.79 \mathrm{~kg}$, and $24.27 \pm 3.05 \mathrm{~kg} / \mathrm{m}^{2}$, respectively. EDS revealed that 40 subjects had CTS in both hands, 7 had CTS only in their right hand, 5 had CTS only in their left hand, and 2 had normal findings.

Table 1 shows the electrophysiologic severities of CTS. 
There was no statistical difference in severity between both hands. In comparing the symptoms, the sensitivity and specificity of EDS were $89.66 \%$ and $33.33 \%$, respectively.

Correlation between the electrophysiologic severity of CTS and the ultrasonographic measurements

The electrophysiologic severity of CTS statistically correlated with weight, BMI, proximal CSA of the median nerve, and proximal CSA of carpal tunnel. There was no relationship between the proximal and distal nerve/tunnel indexes and electrophysiologic severity of CTS (Table 2).

\section{Comparison between the four severity grade groups} by EDS, anthropometric data, and ultrasonographic measurements

There were statistically significant differences in weight, BMI, and proximal CSAs of the median nerve and carpal tunnel among the graded groups (Table 3 ). However, the proximal and distal nerve/tunnel indexes and the distal CSAs of median nerve and carpal tunnel did not show significant differences among these groups.

The cut-off value of ultrasonographic severity according to CSA of median nerve

A previous study suggests that proximal CSA of median nerve could be used for screening CTS in the general population by using US [12]. In line with that study, we conducted ROC analyses in CSA of the proximal median nerve, only. Fig. 3 shows ROC curves of the proximal CSA of the median nerve on the basis of severity grades. Based on the areas under the curve (ACU), we set cut-off values of CSA of median nerve as follows: grade I at $10 \mathrm{~mm}^{2}$ (AUC $=0.66$, sensitivity $=86.52 \%, 1$-specificity $=52.63 \%$ ), grade $\mathrm{II}$ at $11 \mathrm{~mm}^{2}$ (AUC $=0.71$, sensitivity $=82.27 \%$, 1 -specificity $=50.94 \%$ ), and grade III at $12 \mathrm{~mm}^{2}$ (AUC $=0.66$, sensitivity $=86.96 \%$, 1 -specificity $=55.29 \%$ ).

Table 2. Correlation between the severity of carpal tunnel syndrome by electodiagnostic study and anthropometric data, and ultrasonographic measurements

\begin{tabular}{lcc}
\hline & $\begin{array}{c}\text { Spearman } \\
\text { coefficients }\end{array}$ & p-value \\
\hline Age & -0.0118 & 0.93 \\
Height & -0.0752 & 0.59 \\
Weight & 0.3880 & $0.004^{*}$ \\
BMI & 0.4285 & $0.001^{*}$ \\
Proximal CSA of median nerve & 0.3218 & $0.02^{*}$ \\
\hline Proximal CSA of carpal tunnel & 0.3753 & $0.005^{*}$ \\
Proximal nerve/tunnel index & 0.1093 & 0.43 \\
Distal CSA of median nerve & 0.2304 & 0.09 \\
Distal CSA of carpal tunnel & 0.2678 & 0.05 \\
Distal nerve/tunnel index & 0.0528 & 0.71 \\
\hline
\end{tabular}

BMI, body mass index; CSA, cross-sectional area. ${ }^{*} \mathrm{p}<0.05$.

Table 3. Comparison between the graded four groups by categorization of the severity of carpal tunnel syndrome by electrodiagnostic study and anthropometric data, and ultrasonographic measurements

\begin{tabular}{lrrrrc}
\hline & \multicolumn{1}{c}{ Grade 0 } & \multicolumn{1}{c}{ Grade I } & \multicolumn{1}{c}{ Grade II } & \multicolumn{1}{c}{ Grade III } & p-value \\
\hline Age & $51.89(1.96)$ & $55.82(1.47)$ & $54.06(1.51)$ & $52.00(1.79)$ & 0.28 \\
Height & $158.11(0.89)$ & $156.74(0.66)$ & $157.75(0.69)$ & $156.65(0.81)$ & 0.46 \\
Weight & $55.47(1.68)$ & $59.09(1.26)$ & $60.66(1.29)$ & $64.35(1.53)$ & $0.002^{*}$ \\
BMI & $22.19(0.64)$ & $24.04(0.48)$ & $24.37(0.50)$ & $26.22(0.58)$ & $0.002^{*}$ \\
Proximal CSA of median nerve $\left(\mathrm{mm}^{2}\right)$ & $11.08(1.03)$ & $12.03(0.77)$ & $13.93(0.79)$ & $15.20(0.94)$ & $0.010^{*}$ \\
Proximal CSA of carpal tunnel $\left(\mathrm{mm}^{2}\right)$ & $174.68(9.12)$ & $182.74(6.81)$ & $202.47(7.02)$ & $218.09(8.29)$ & $0.001^{*}$ \\
Proximal nerve/tunnel index $(\%)$ & $6.48(0.53)$ & $6.74(0.40)$ & $7.02(0.41)$ & $7.22(0.48)$ & 0.73 \\
Distal CSA of median nerve $\left(\mathrm{mm}^{2}\right)$ & $10.31(0.66)$ & $10.02(0.50)$ & $10.88(0.51)$ & $12.02(0.60)$ & 0.07 \\
Distal CSA of carpal tunnel $\left(\mathrm{mm}^{2}\right)$ & $136.89(5.23)$ & $137.94(3.91)$ & $144.78(4.03)$ & $152.87(4.75)$ & 0.06 \\
Distal nerve/tunnel index $(\%)$ & $7.63(0.46)$ & $7.32(0.34)$ & $7.65(0.36)$ & $7.85(0.42)$ & 0.80 \\
\hline Values & & & & &
\end{tabular}

Values are presented as mean (standard error).

BMI, body mass index; CSA, cross-sectional area.

${ }^{*} \mathrm{p}<0.05$. 


\section{DISCUSSION}

This study revealed that electrophysiologic severity of CTS significantly correlated to ultrasonographic indexes (such as proximal CSAs of the median nerve and carpal tunnel), as well as body indexes (such as weight and BMI) in female CTS patients. In addition, we found the proximal CSA of the median nerve, which a previous study used as a screening tool for examining CTS [12], could provide the cut-off value for each severity grade of CTS in those patients. On the other hand, the proximal and distal nerve/tunnel indexes were constant regardless of the EDS results.

Until now, EDS has been the main tool for diagnosing CTS. However, EDS has some undesirable consequences, such as invasiveness, painfulness, and limitations in searching the surrounding tissues. In addition, in severe cases, EDS has difficulty in confirming diagnosis due to thenar muscle atrophy. Conversely, using US is easier for detecting marked nerve enlargement in cases of paralyzed APB and thenar muscle atrophy [19].

For these reasons, studies have focused on ultrasonographic screening methods for CTS in the general population. Previous studies show that CSA of the proximal median nerve is $9.0 \mathrm{~mm}^{2}$ [12]. However, that value is different from our study $\left(10 \mathrm{~mm}^{2}\right)$ Such a difference could arise from different study settings, such as the diagnostic method of EDS, environment of EDS, gender distinctions, and race.

In taking a step forward, we tried to provide a cut-off value for each severity grade in female CTS patients. By using cut-off values of proximal CSA of the median nerve in an ultrasonographic exam, clinicians could predict the severity of CTS in female patients, and get further information about surrounding tissues without invasiveness before EDS.

In a previous study, normal subjects without any symptoms of CTS revealed that ultrasonographic measurements of the proximal and distal CSAs of median nerve and carpal tunnel were greater in males than females, and proximal and distal CSAs of the median nerve and carpal tunnel were related to BMI [5]. In another study, the proximal CSA of the median nerve and the proximal nerve/tunnel index of the affected hands were significantly greater than those of the non-affected hands, but only in females [8]. In view of these points, we found it necessary to distinguish the gender of the patients to study the ultrasonographic indexes in CTS. If the same ultrasonographic diagnostic criteria were applied to both genders, a false-positive rate would increase in men and a false-negative rate would increase in women [8]. In addition, previous studies had not proven the correlation between the ultrasonographic index and electrodiagnostic severity in CTS $[12,20]$. These studies had not considered the difference of gender and body indexes. Thus, in this study, proximal CSAs of median nerve and carpal tunnel confirmed a statistically significant correlation with electrodiagnostic severity. Thus, we thought that the most important factor in these different results is the distinction between patients according to gender.

The carpal tunnel area is surrounded by carpal bones and rigid ligament, which contain the median nerve and nine tendons. Any event resulting in increased pressure of the carpal tunnel area may cause CTS. The pathophysiology and etiology of CTS are being continuously and extensively investigated [3,9,21-23]. In these studies, many probable causes point to the major pathophysiology of CTS, like space occupying lesion, finger flexor tenosynovitis, and idiopathic origin. However, there are disagreements on the primary etiologies of CTS. We used ultrasonographic measurements, including nerve/tunnel index and CSA of median nerve and carpal tunnel in proximal and distal, on diagnosing and predicting severity of CTS. With these measurements, we sought to provide more information on explaining the mechanism of CTS, even though the exact pathophysiology is still unkonwn.

In a separate study, the median nerve was mainly compressed around the distal carpal tunnel rather than the proximal carpal tunnel [8]. In consideration of the results of our study, the idiopathic pressing of the median nerve at the distal carpal tunnel and swelling of median nerve at the proximal carpal tunnel could be the reason for CTS, considering the enlarged proximal CSA of the median nerve and carpal tunnel.

In this study, the proximal CSA of carpal tunnel correlated to electrophysiologic severity of CTS. Many other studies used US in diagnosing CTS and studying the relationship between electrodiagnostic severity. However most of these other studies only used the value of the CSA of median nerve at the proximal level other than CSA of carpal tunnel $[10,19,24]$. Usually, a good spatial resolution of US allows physicians to easily detect the swelling 
of the median nerve in a long-axis view of CTS, apart from the absolute value of median nerve area. However, it is difficult to detect the successive changes of the flexor tendon around carpal tunnel inlet and outlet, except in the mass-occupying lesions of CTS. In addition, it is easier to measure CSA of median nerve than that of carpal tunnel, and it requires more time to trace the boundary of carpal tunnel than that of the median nerve. For these reasons, we focused on the proximal CSA of the median nerve to derive a cut-off value to discriminate the severity of CTS in females.

This study has some limitations. Hand temperature measurements were only performed before starting EDS, not continuously. Nerve conduction velocity is greatly affected by body temperature and continuous hand monitoring during EDS is recommended [25]. However, although not ideal, we did perform the hand temperature measurement before the start of EDS and maintained a constant room temperature during examination.

Another limitation is our lack of data on the correlation between BMI and CSAs of median nerve and carpal tunnel. Previous studies also showed a significant correlation, but the statistical correlation was not made in our study.

In conclusion, our study showed that electrodiagnostic severity correlates with ultrasonographic severity in females. Therefore, suggested cut-off values by ultrasonographic severity level of CTS. We only enrolled females for this study. Therefore, we cannot apply the results of this study to males. A follow-up study with a substantial number of male patients is necessary to set up the severity level in males.

\section{CONFLICT OF INTEREST}

No potential conflict of interest relevant to this article was reported.

\section{ACKNOWLEDGMENTS}

The authors would like to thank Seong Woo Kim, MD for interpretation of the electrodiagnostic results.

\section{REFERENCES}

1. Karadag YS, Karadag O, Cicekli E, Ozturk S, Kiraz S,
Ozbakir S, et al. Severity of carpal tunnel syndrome assessed with high frequency ultrasonography. Rheumatol Int 2010;30:761-5.

2. de Krom MC, Knipschild PG, Kester AD, Thijs CT, Boekkooi PF, Spaans F. Carpal tunnel syndrome: prevalence in the general population. J Clin Epidemiol 1992;45:373-6.

3. Duncan I, Sullivan P, Lomas F. Sonography in the diagnosis of carpal tunnel syndrome. AJR Am J Roentgenol 1999;173:681-4.

4. Pastare D, Therimadasamy AK, Lee E, Wilder-Smith EP. Sonography versus nerve conduction studies in patients referred with a clinical diagnosis of carpal tunnel syndrome. J Clin Ultrasound 2009;37:389-93.

5. Kim HS, Joo SH, Han ZA, Kim YW. The nerve/tunnel index: a new diagnostic standard for carpal tunnel syndrome using sonography: a pilot study. J Ultrasound Med 2012;31:23-9.

6. Carneiro RS, Velasquez L, Tietzman A. Trigger wrist caused by a tumor of the tendon sheath in a teenager. Am J Orthop (Belle Mead NJ) 2001;30:233-4.

7. Bou-Merhi JS, Harris PG, Brutus JP. "Trigger finger at the wrist" due to anomalous flexor digitorum superficialis muscle belly within the carpal tunnel. Chir Main 2007;26:238-42.

8. Kim HS, Joo SH, Cho HK, Kim YW. Comparison of proximal and distal cross-sectional areas of the median nerve, carpal tunnel, and nerve/tunnel index in subjects with carpal tunnel syndrome. Arch Phys Med Rehabil 2013;94:2151-6.

9. El Miedany YM, Aty SA, Ashour S. Ultrasonography versus nerve conduction study in patients with carpal tunnel syndrome: substantive or complementary tests? Rheumatology (Oxford) 2004;43:887-95.

10. Moran L, Perez M, Esteban A, Bellon J, Arranz B, del Cerro M. Sonographic measurement of cross-sectional area of the median nerve in the diagnosis of carpal tunnel syndrome: correlation with nerve conduction studies. J Clin Ultrasound 2009;37:125-31.

11. Claes F, Kasius KM, Meulstee J, Verhagen WI. Comparing a new ultrasound approach with electrodiagnostic studies to confirm clinically defined carpal tunnel syndrome: a prospective, blinded study. Am J Phys Med Rehabil 2013;92:1005-11.

12. Mhoon JT, Juel VC, Hobson-Webb LD. Median nerve ultrasound as a screening tool in carpal tunnel syn- 
drome: correlation of cross-sectional area measures with electrodiagnostic abnormality. Muscle Nerve 2012;46:871-8.

13. Tai TW, Wu CY, Su FC, Chern TC, Jou IM. Ultrasonography for diagnosing carpal tunnel syndrome: a metaanalysis of diagnostic test accuracy. Ultrasound Med Biol 2012;38:1121-8.

14. Fowler JR, Gaughan JP, Ilyas AM. The sensitivity and specificity of ultrasound for the diagnosis of carpal tunnel syndrome: a meta-analysis. Clin Orthop Relat Res 2011;469:1089-94.

15. American Association of Electrodiagnostic Medicine; American Academy of Neurology; American Academy of Physical Medicine and Rehabilitation. Practice parameter for electrodiagnostic studies in carpal tunnel syndrome: summary statement. Muscle Nerve 1993; 16:1390-1.

16. Uncini A, Lange DJ, Solomon M, Soliven B, Meer J, Lovelace RE. Ring finger testing in carpal tunnel syndrome: a comparative study of diagnostic utility. Muscle Nerve 1989;12:735-41.

17. Werner RA, Andary M. Electrodiagnostic evaluation of carpal tunnel syndrome. Muscle Nerve 2011;44:597607.

18. Bland JD. A neurophysiological grading scale for carpal tunnel syndrome. Muscle Nerve 2000;23:1280-3.

19. Nakamichi K, Tachibana S. Ultrasonographic mea- surement of median nerve cross-sectional area in idiopathic carpal tunnel syndrome: diagnostic accuracy. Muscle Nerve 2002;26:798-803.

20. Yazdchi M, Tarzemani MK, Mikaeili H, Ayromlu H, Ebadi H. Sensitivity and specificity of median nerve ultrasonography in diagnosis of carpal tunnel syndrome. Int J Gen Med 2012;5:99-103.

21. Dumitru D, Amato AA, Zwarts M. Electrodiagnostic medicine. 2nd ed. Philadelphia: Hanley \& Belfus; 2002.

22. Buchberger W, Judmaier W, Birbamer G, Lener M, Schmidauer C. Carpal tunnel syndrome: diagnosis with high-resolution sonography. AJR Am J Roentgenol 1992;159:793-8.

23. Bianchi S, Martinoli C. Ultrasound of the musculoskeletal system. Heidelberg: Springer; 2007.

24. Yesildag A, Kutluhan S, Sengul N, Koyuncuoglu HR, Oyar O, Guler K, et al. The role of ultrasonographic measurements of the median nerve in the diagnosis of carpal tunnel syndrome. Clin Radiol 2004;59:910-5.

25. Jablecki CK, Andary MT, Floeter MK, Miller RG, Quartly CA, Vennix MJ, et al. Practice parameter: electrodiagnostic studies in carpal tunnel syndrome. Report of the American Association of Electrodiagnostic Medicine, American Academy of Neurology, and the American Academy of Physical Medicine and Rehabilitation. Neurology 2002;58:1589-92. 